Sangeeta K. Siri* and Mrityunjaya V. Latte

\title{
A Novel Approach to Extract Exact Liver Image Boundary from Abdominal CT Scan using Neutrosophic Set and Fast Marching Method
}

https://doi.org/10.1515/jisys-2017-0144

Received April 9, 2017; previously published online August 22, 2017.

\begin{abstract}
Liver segmentation from abdominal computed tomography (CT) scan images is a complicated and challenging task. Due to the haziness in the liver pixel range, the neighboring organs of the liver have the same intensity level and existence of noise. Segmentation is necessary in the detection, identification, analysis, and measurement of objects in CT scan images. A novel approach is proposed to meet the challenges in extracting liver images from abdominal CT scan images. The proposed approach consists of three phases: (1) preprocessing, (2) CT scan image transformation to neutrosophic set, and (3) postprocessing. In preprocessing, noise in the CT scan is reduced by median filter. A "new structure" is introduced to transform a CT scan image into a neutrosophic domain, which is expressed using three membership subsets: true subset $(T)$, false subset $(F)$, and indeterminacy subset $(I)$. This transform approximately extracts the liver structure. In the postprocessing phase, morphological operation is performed on the indeterminacy subset $(I)$. A novel algorithm is designed to identify the start points within the liver section automatically. The fast marching method is applied at start points that grow outwardly to detect the accurate liver boundary. The evaluation of the proposed segmentation algorithm is concluded using area- and distance-based metrics.
\end{abstract}

Keywords: Neutrosophic set, fast marching method, computed tomography, image processing, liver segmentation.

\section{Introduction}

The liver is a vital organ that has many roles in the body, including building proteins and blood clotting, manufacturing triglycerides and cholesterol, glycogen synthesis, and bile production. The liver is the largest internal organ. Infections such as hepatitis, cirrhosis (scarring), cancer, and the overeffects of medications are identified diseases within the liver. The foremost stage for the computer-aided diagnosis of the liver is the identification of the liver region. Liver segmentation algorithms extract liver images from scan images, which helps in virtual surgery simulation and speeds up the disease diagnosis, accurate investigation, and surgery planning.

Liver segmentation from computed tomography (CT) scan images has gained a lot of importance in the medical image processing field because 1 in every 94 men and 1 in every 212 women born are susceptible to liver cancer in their lifetime [3, 14]. Liver cancer is one of the most common diseases, with increasing morbidity and high mortality $[9,33]$. Liver cancer treatment requires maximum radiation dose to the tumor and minimum toxicity to the surrounding healthy tissues. This is the major challenge in clinical practice [25, 32]. Selective internal radiation therapy (SIRT) with yttrium-90 (Y-90) microspheres is an effective technique for liver-directed therapy [15]. SIRT dosimetry requires an accurate determination of the relative functional

*Corresponding author: Sangeeta K. Siri, Department of Electronics and Communication Engineering, Sapthagiri College of Engineering, Bengaluru, Karnataka, India, e-mail: sangeeta_mk@rediffmail.com

Mrityunjaya V. Latte: JSS Academy of Technical Education, Bengaluru, Karnataka, India 
tumor(s) volume(s) with respect to the anatomical volumes of the liver to estimate the necessary Y-90 microsphere dose [4, 23]. Clinically, accurate liver volume determination is accomplished through tedious manual segmentation of the entire CT scan. A task is greatly dependent on the skill of the operator. Manual segmentation is time consuming. Thus, many automatic or semiautomatic techniques are available for segmentation and determine the volume of the liver accurately. This facilitates the operational process from a physician's viewpoint.

Extracting liver from CT or magnetic resonance imaging (MRI) scan images is of prime importance. Considerable work has been done in extracting liver images from CT or MRI scan images, so a general solution has remained a challenge. Failure in getting a reliable and accurate segmentation algorithm is due to the following reasons: (1) The neighboring organs of the liver, such as the kidneys, heart, and stomach, have the same intensity level. (2) There is no definite shape, weight, size, volume, or texture for a liver. All these parameters are subjective. (3) The edges are weak. (4) The presence of artifacts in MRI or CT scan images. (5) Variability of liver geometry from patient to patient. (6) Large variation in pixel level range throughout the liver section as well as from patient to patient.

\section{Related Work}

Chen et al. [6] designed the Chan-Vese (C-V) model for liver segmentation in which the Gaussian function is used to find liver likelihood images from CT scan images and obtain the liver boundary using the C-V model. They have used morphological operation to improve the results. Song et al. [31] proposed an automatic liver boundary marking method based on an adaptive fast marching method (FMM). The liver image is separated from the CT scan by manually fixing the pixel intensity between 50 and 200. Median filter is applied to reduce noise and the liver image is enhanced by sigmoidal function. In this, the image is converted into binary and FMM is applied to find liver boundary accurately. Wu et al. [33] developed a novel method for the automatic delineation of the liver on CT volume images using supervoxel-based graph cuts. This method integrates histogram-based adaptive thresholding, simple linear iterative clustering, and graph cuts algorithm. Moghbel et al. [21] proposed random walker-based framework. In this, the liver dome is automatically detected based on the location of the right lung lobe and ribcage area and the liver is extracted using the random walker method. Ding et al. [7] introduced a multiatlas segmentation approach with local decision fusion for fast automated liver (with/without abnormality) segmentation on CT angiography. Zheng et al. [37] designed a feature learning-based random walker method for liver segmentation using CT images. Four texture features are extracted and then classified to determine the probability corresponding to the test images. In this, seed points on the original test image are automatically selected. Peng et al. [24] designed a novel multiregion appearance-based approach with graph cuts to delineate the liver surface and a geodesic distance-based appearance selection scheme is introduced to use proper appearance constraint for each subregion. Platero and Tobar [26] proposed a new approach to segment the liver from the CT scan, which is the combination of low-level operations, an affine probabilistic atlas, and a multiatlas-based segmentation. Salman AlShaikhli et al. [27] presented a novel fully automatic algorithm for 3D liver segmentation in clinical 3D CT images based on the Mahalanobis distance cost function using an active shape model implemented on MICCAI-SLiver07 achieving accurate results. Lu et al. [19] developed liver segmentation using a 3D convolutional neural network and the accuracy of initial segmentation is increased with graph cut algorithm and the previously learned probability map. Li et al. [18] developed a technique to detect the liver surface, which includes the construction of a statistical shape model using principal component analysis. Euclidean distance transformation is used to obtain a coarse position in a source image. The accurate detection of the liver is obtained using the deformable graph cut method. Zheng et al. [36] designed a tree-like multiphase level set algorithm for segmentation based on the $\mathrm{C}-\mathrm{V}$ model to detect objects in an image. The algorithm is effective for images that have subobjects in the region. 


\section{Neutrosophic Set (NS)}

NS was introduced by Samarandache [28]. In the NS theory, every event has not only a certain degree of the truth but also a falsity degree with indeterminacy. These parameters are considered independent from each other $[13,22]$. An entity $\{S\}$ is considered with opposite $\{$ Anti-S $\}$ and neutrality $\{$ Neut-S\}. The $\{$ Neut-S\} and $\{$ Anti-S\} are referred to as $\{$ Non-S $[1,10,11,22]$.

To apply the concept of NS to image processing, the image should be transformed into the neutrosophic domain. Image $P$ of size $X^{\star} Y$ with $K$ gray levels can be defined as three arrays of neutrosophic images described by three membership sets: $T$ (true subset), $I$ (indeterminate subset), and $F$ (false subset). Therefore, a pixel $P(i, j)$ in the image transferred into the neutrosophic domain can be represented by $P_{\mathrm{NS}}=\{T(i, j), I(i, j)$, $F(i, j)\}$ or $P_{\mathrm{NS}}=P(t, i, f)$. It means that the pixel is $\% t$ true, $\% i$ indeterminate, and $\% f$ false [29]. Here, $t$ varies in $T$ (white pixel set), $i$ varies in $I$ (noise pixel set), and $f$ varies in $F$ (black pixel set), which are defined as follows $[22,28]:$

$$
\begin{gathered}
T(i, j)=\frac{\bar{G}(i, j)-\bar{G}_{\text {min }}}{\bar{G}_{\text {max }}-\bar{G}_{\text {min }}} \\
I(i, j)=\frac{d(i, j)-d_{\text {min }}}{d_{\text {max }}-d_{\text {min }}} \\
F(i, j)=1-T(i, j)
\end{gathered}
$$

where $\bar{G}(i, j)$ is the local mean value of the pixel of the window and given by following equation:

$$
\bar{G}(i, j)=\frac{1}{w^{\star}} w_{m=i-w / 2}^{m=i+w / 2} \sum_{n=i-w / 2}^{j+w / 2} G(m, n)
$$

$d(i, j)$ is the absolute value of the difference between intensity $G(i, j)$ and its local mean value $\bar{G}(i, j)$ and given as

$$
d(i, j)=\operatorname{abs}(G(i, j)-\bar{G}(i, j))
$$

$G(i, j)$ is the intensity value of the pixel $P(i, j) ; w$ is the size of the sliding window; $\bar{G}_{\min }$ and $\bar{G}_{\text {max }}$ are the minimum and maximum of the local mean values of the image, respectively; and $d_{\min }$ and $d_{\max }$ are the minimum and maximum value of $d(i, j)$ in the whole image.

\section{FMM}

In the intended method, Sethian's FMM [30] has been used to track the evolution of an expanding front. In our case, a front is a closed 2D curve that separates the interior and exterior regions. Because the closed surface is a curve in 2D and the lattice is a pixel raster, the FMM assigns to the pixel the time at which the expanding curve hits the pixel. Hence, at a given point, the motion of the front is described by the equation known as the Eikonal equation [2, 30, 31]:

$$
|\nabla V(x)|=\frac{1}{S(x)} x \in R
$$

where $V(x)$ is the arrival time of the front at point $x$ and $S(x)$ is the speed function of front at point $x$. Because the front cannot only be expanded, the arrival time $V$ is single valued. We assume that $S=0$ everywhere. 
The FMM simply propagates the shortest distance to the boundary from starting point. The above Eikonal equation is discretized and solved with the upwind scheme. For 2D grids, $S$ can be obtained as follows:

$$
\left[\max \left(d^{-x} V, 0\right)^{2}+\min \left(d^{+x} V, 0\right)^{2}+\max \left(d^{-y} V, 0\right)^{2}+\min \left(d^{+y} V, 0\right)^{2}\right]=1 / S
$$

where $d^{+}$and $d^{-}$are the standard forward and backward operators, respectively. We have taken $[10 ;-10 ; 01$; $0-1]$ as the difference operator.

\section{Methodology}

\subsection{Preprocessing Phase}

The abdominal CT scan image is with a $1019 \times 682$ DICOM color format. First, convert the CT scan image into a grayscale image of size $512 \times 512$. Reduce the noise using median filter.

\subsection{Map the CT Scan Image into NS Domain}

Step 1: Crop random section of the liver image.

Step 2: Obtain true subset $T$ and false subset $F$ using bell function.

$$
T(x, y)=\pi\left(C_{x y}, a, b, c, d\right)=\left\{\begin{array}{cc}
0 & 0 \leq C_{x y}<a \\
\frac{\left(C_{x y}-a\right)^{2}}{(d-a)(d-a)} & a \leq C_{x y}<b \\
1-\frac{\left(C_{x y}-b\right)}{(d-c)(d-c)} & b \leq C_{x y} \leq c \\
\frac{\left(C_{x y}-d\right)^{2}}{(d-c)(d-c)} & c<C_{x y} \leq d \\
0 & C_{x y}>d \\
F(x, y)=1-T(x, y) &
\end{array}\right.
$$

where $C_{x y}$ is the intensity value of pixel $(i, j)$ in the cropped image of the liver. Variables $a-d$ are the parameters that determine the shape of the bell function as shown in Figure 1.

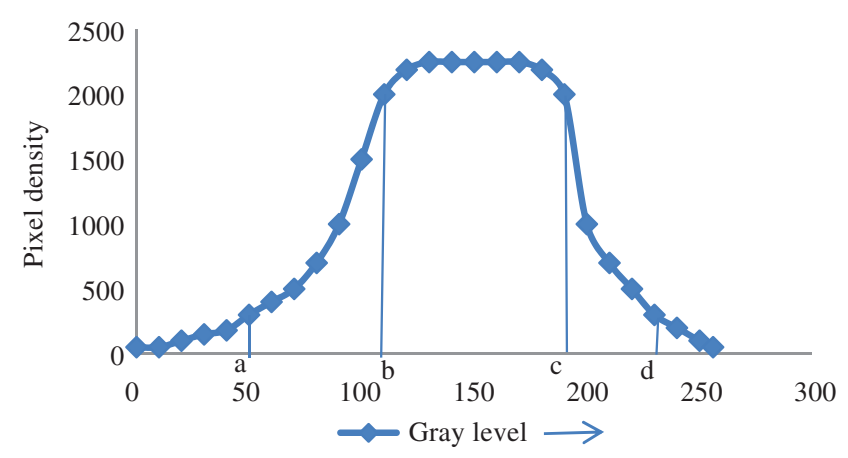

Figure 1: Bell Function. 
The values of variables $a-d$ are obtained using the histogram-based method as follows:

(i) Obtain the histogram of the cropped liver section.

(ii) Find the local maxima of the histogram.

$$
P_{\max }\left(g_{1}\right), P_{\max }\left(g_{2}\right), P_{\max }\left(g_{3}\right), \ldots, P_{\max }\left(g_{n}\right) .
$$

(iii) Calculate the mean of local maxima.

$$
\bar{P}_{\max }=\frac{\sum_{i=1}^{n} P_{\max }\left(g_{i}\right)}{n}
$$

(iv) Find the peak values greater than the mean of local maxima $P_{\max }$.

$b$ is the initial peak value; $c$ is the final peak value.

(v) Find the standard deviation (SD) of the cropped section of the liver.

where $\bar{x}=\frac{1}{n} \sum_{i=1}^{n} x_{i}$

$$
\mathrm{SD}=\left(\frac{1}{n} \sum_{i=1}^{n}\left(x_{i}-\bar{x}\right)^{2}\right)^{1 / 2}
$$

(vi) Find the value of $a$ and $d$ as follows:

$$
\begin{aligned}
& a=b-\mathrm{SD} \\
& d=c+\mathrm{SD}
\end{aligned}
$$

Step 3: Convert $T$ and $F$ into binary [34].

$T_{\text {th }}$ and $F_{\text {th }}$ are the thresholds in true subset $(T)$ and false subset $(F)$, respectively. These are also required to obtain the indeterminacy subset (I). A heuristic approach is used to find the thresholds in $T$ and $F$.

(i) Select an initial threshold $t_{0}$ in $T$.

(ii) Separate $T$ using $t_{0}$ and obtain two new groups of pixels: $T 1$ and $T 2$. ( $\mu 1$ and $\mu 2$ are the mean values of these two groups.)

(iii) Compute new threshold value $t_{1}=\frac{\mu 1+\mu 2}{2}$.

(iv) Repeat steps (ii)-(iv) until the difference of $t_{n}-t_{n-1}$ is smaller than $\varepsilon$ ( $\varepsilon=0.001$ in the experiments) in successive iterations. Then, threshold $T_{\text {th }}$ is calculated by following substitution:

$$
T_{\text {th }}=t_{n}
$$

(v) The above steps are repeated for finding $F_{\text {th }}$ in false subset $(F)$.

Step 4: Find the indeterminacy subset $(I)[34,35]$.

Homogeneity is related to local information and plays an important role in image segmentation. We can define homogeneity using the SD and discontinuity of the intensity. SD describes the contrast within a local region, whereas discontinuity represents the changes in gray levels. Objects and background are more uniform and blurry edges are gradually changing from objects to background. The homogeneity value of objects and background is larger than that of the edges. A randomly identified size $D \times D$ window centered at $(x, y)$ is used for computing the SD of pixel $(i, j)$ :

$$
S_{d}=\sqrt{\frac{\sum_{p=x-(D-1) / 2}^{x+(D-1) / 2} \sum_{q=y-(D-1) / 2}^{y+(D-1) / 2}\left(G_{x y}-\mu_{x y}\right)^{2}}{D^{2}}}
$$

where $\mu_{x y}$ is the mean of intensity values within the window and given by following equation:

$$
\mu_{x y}=\frac{\sum_{p=x-(D-1) / 2}^{x+(D-1) / 2} \sum_{q=y-(D-1) / 2}^{y+(D-1) / 2} G_{x y}}{d^{2}}
$$


The discontinuity of pixel $P(i, j)$ is described by the edge value. We use Sobel operator to calculate the discontinuity.

$$
E_{g}(x, y)=\sqrt{G_{x}^{2}+G_{y}^{2}}
$$

where $G_{x}$ and $G_{y}$ are the horizontal and vertical derivative approximations.

Normalize the SD and discontinuity and define the homogeneity as

$$
H(x, y)=1-\frac{S_{d}(x, y)}{S_{d \max }} \star \frac{E_{g}(x, y)}{E_{g \max }}
$$

where $S_{d \max }=\max \left\{S_{d}(x, y)\right\}$ and $E_{g \max }=\max \left\{E_{g}(x, y)\right\}$

The indeterminacy $I(x, y)$ is represented as

$$
I(x, y)=1-H(x, y)
$$

The value of $I(x, y)$ has a range of $0-1$. The more uniform the region surrounding a pixel results in the minimum value of the indeterminate pixel. The window size should be big enough to include enough local information but still be less than the distance between the two objects. We chose $D=10$ in all calculations.

Step 5: Convert $T, F$, and $I$ into binary image.

(a) Procedure to find value of $\propto$.

- $\min =$ minimum $\{$ maximum values of each column in indeterminacy image $(I) \neq 0\}$.

- $\propto$ is any value less than or equal to min.

(b) In this step, a given image is divided into three parts: object (Obj), edge (Edge), and Background (Bkg). $T(x, y)$ represents the degree of being an object pixel (Obj), $I(x, y)$ is the degree of being an edge pixel (Edge), and $F(x, y)$ is the degree of being a background pixel (Bkg) for pixel $P(x, y)$. The three parts are defined as follows:

$$
\begin{aligned}
& \text { Obj }(x, y)= \begin{cases}\text { True } & T(x, y) \geq T_{\text {th }}, I(x, y)<\propto \\
\text { False } & \text { Others }\end{cases} \\
& \text { Edge }(x, y)= \begin{cases}\text { True } & T(x, y)<T_{\text {th }} \vee F(x, y)<F_{\text {th }}, I(x, y) \geq \propto \\
\text { False } & \text { Others }\end{cases} \\
& \operatorname{Bkg}(x, y)= \begin{cases}\text { True } & F(x, y) \geq F_{\text {th }}, I(x, y)<\infty \\
\text { False } & \text { Others }\end{cases}
\end{aligned}
$$

The objects and background are mapped to 0 and the edges are mapped to 1 in the binary image. The mapping function is as follows:

$$
\operatorname{Binary}(x, y)= \begin{cases}0 & \operatorname{Obj}(x, y) \vee \operatorname{Bkg}(x, y) \vee \overline{\operatorname{Edge}(x, y)}=\text { True } \\ 1 & \text { Others }\end{cases}
$$

\subsection{Postprocessing Phase}

Step 1: Perform morphological operation on indeterminacy set $(I)$. This is called the speed function.

Step 2: Initial contour identification. FMM requires an initial contour from which the evolution of contour starts to detect the object boundary. In this paper, an effective initialization approach for the segmentation of the liver for FMM is proposed. The following steps are designed. 


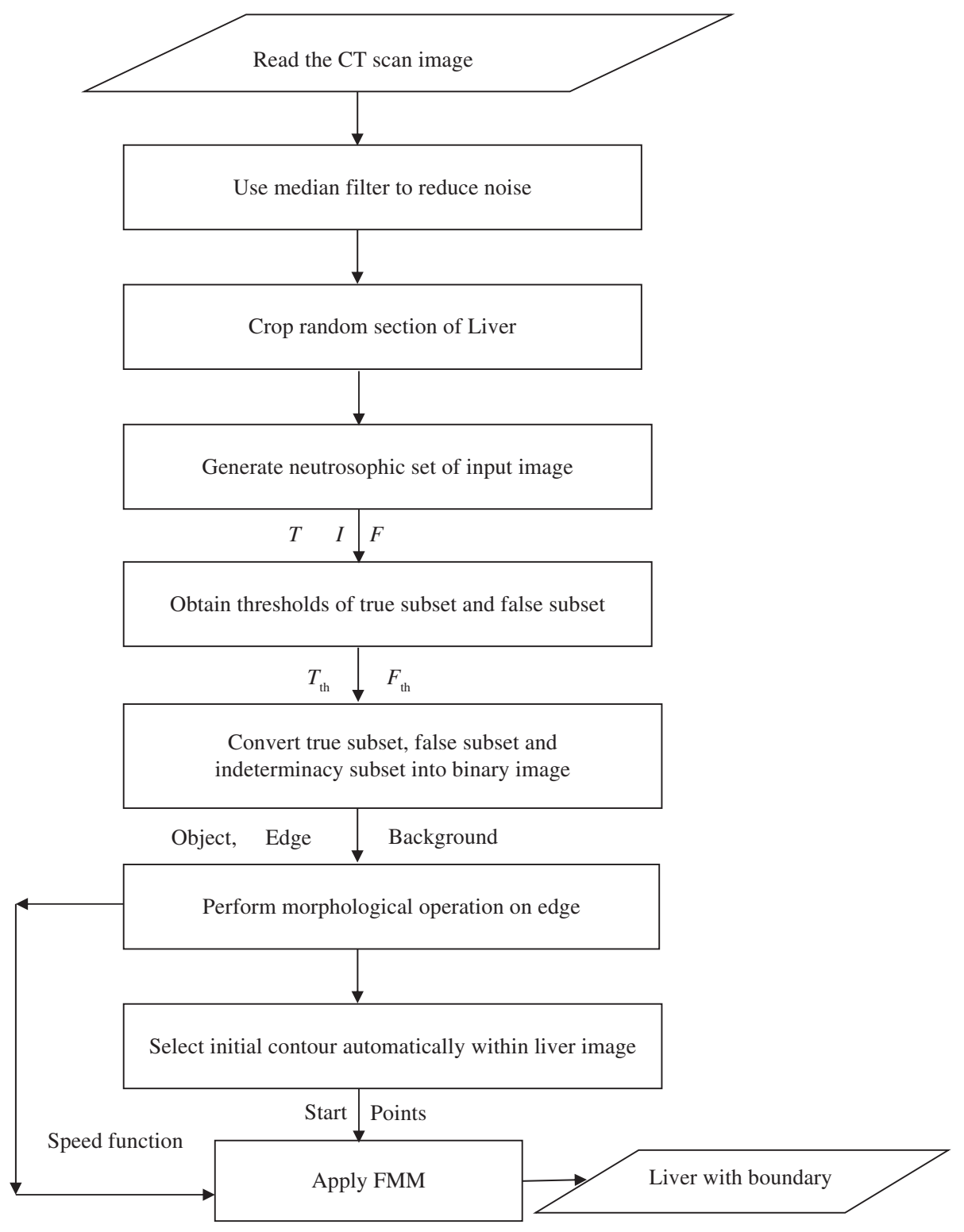

Figure 2: Flowchart of the Proposed Liver Segmentation Algorithm.

(a) Find out the centroid of the liver as (centx', centy').

(b) Choose diff $=20$, which is chosen experimentally.

(c) The Points (centx', centy'), (centx' + diff, centy'), (centx', centy' + diff), (centx' + diff, centy' + diff) positioned in the liver are chosen as the start points. These four start points are located within the liver section.

Step 3: Apply FMM, which detects the liver boundary in the CT scan.

The complete process of the proposed technique is depicted in Figure 2.

\section{Experimental Results}

The experimental data set contains 108 patients' CT scan images, which are provided by M/S CT Scan Centre, Hubli, Karnataka, India. Each slice of the CT scan is a $1019 \times 682$ size color image. 
The original CT scan image, filtered image, and cropped random section of the liver are shown in Figure 3A-C, respectively. The true subset, false subset, and indeterminacy subset are shown in Figure 3D-F, respectively. The object image, background image, and edge images are shown in Figure 3G-I, respectively. The homogeneity image, speed function, and initial contour within the liver section are shown in Figure 3J-L, respectively. The final liver boundary at final iteration, extraction of the liver from abdominal CT scan, and boundary of the liver in the CT scan image are shown in Figure 3M-O, respectively. The ground truth image is shown in Figure 3P.

Figure 4 illustrates the experimental results of the proposed method for four images: columns (a) input image (CT scan image), (b) edge image of the CT scan, (c) speed function of the liver image, (d) liver with boundary, and (e) ground truth image.
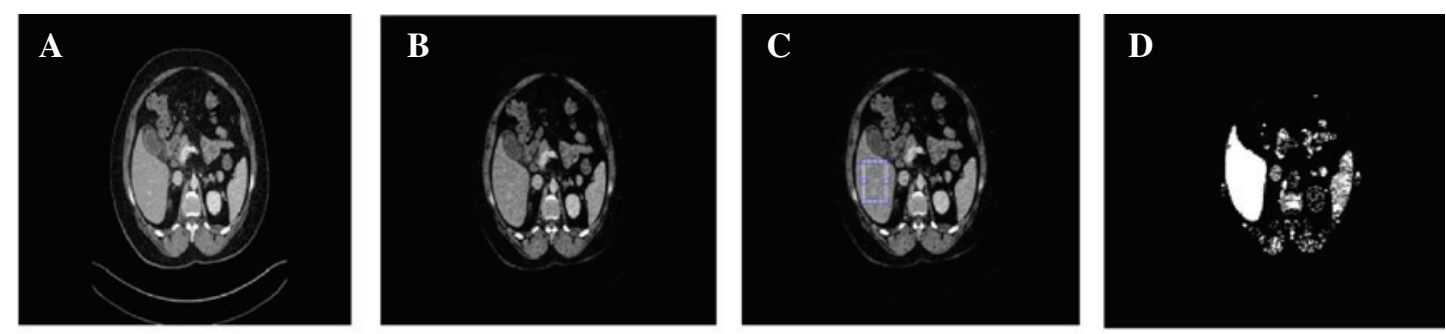

$\mathbf{E}$
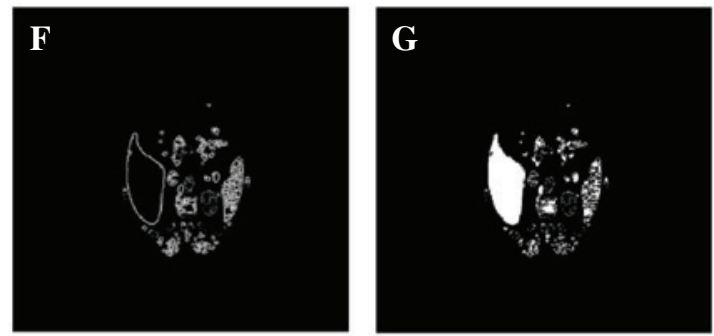

H

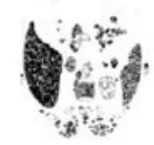

$\mathbf{J}$
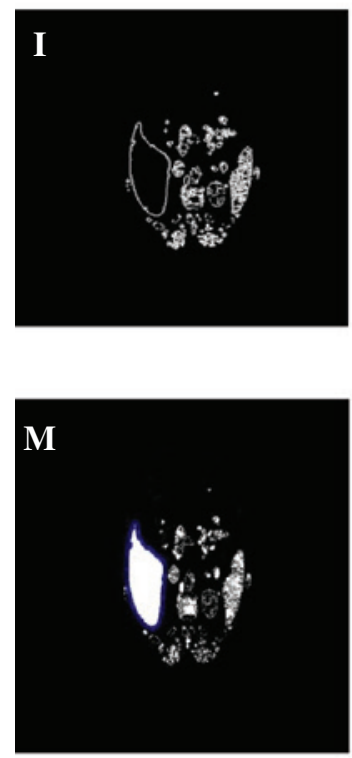
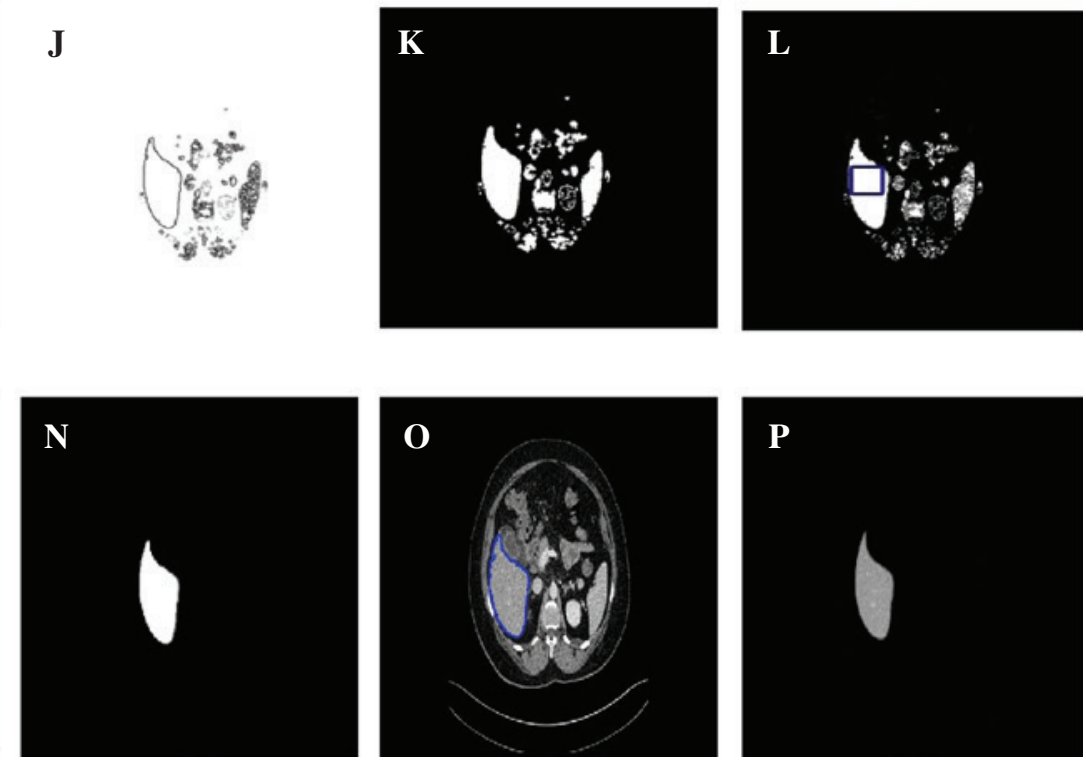

Figure 3: Illustration of liver extraction from CT scan using proposed method.

(A) Original CT scan image, (B) median filter image, (C) cropped random section of the liver, (D) true subset image, (E) false subset image, (F) indeterminate image, (G) foreground image, (H) background image, (I) edge image, (J) homogeneity image, (K) speed function, $(\mathrm{L})$ initial contour in speed function, $(\mathrm{M})$ liver boundary, $(\mathrm{N})$ extraction of the liver from the CT scan image, (O) liver with boundary in CT scan and $(\mathrm{P})$ ground truth image. 
A
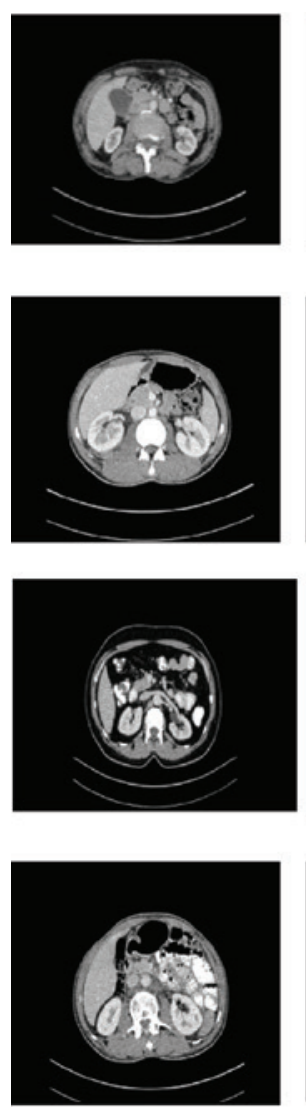

B
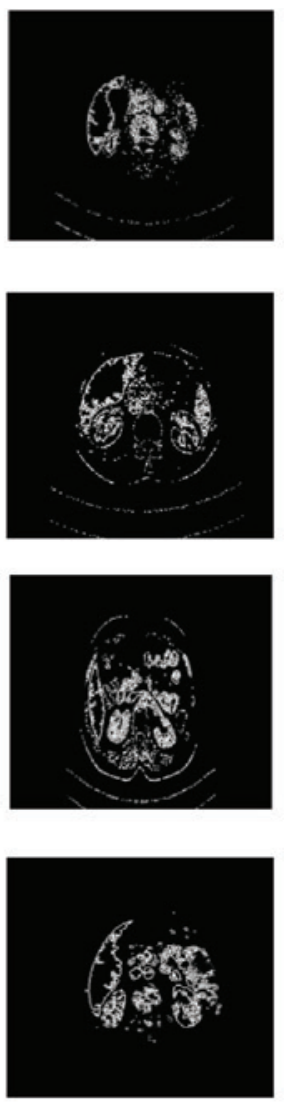

C
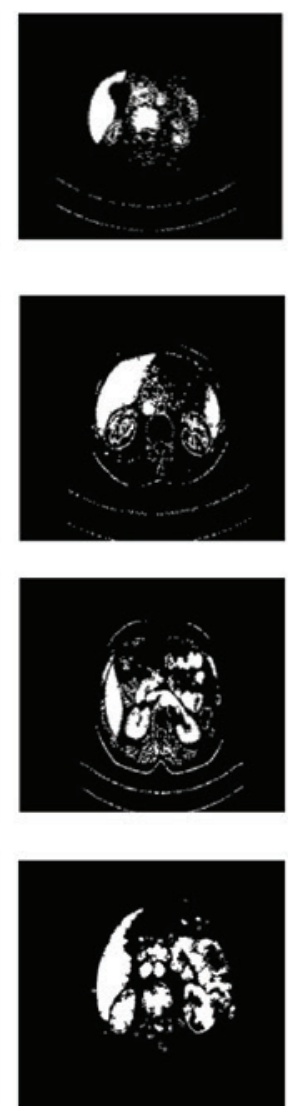

D
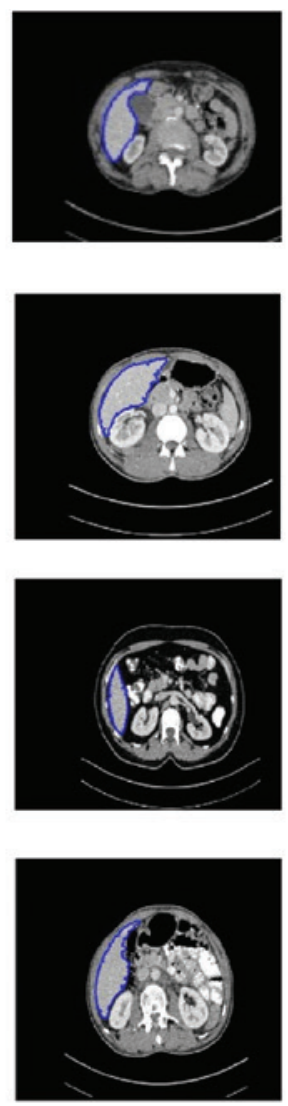

E
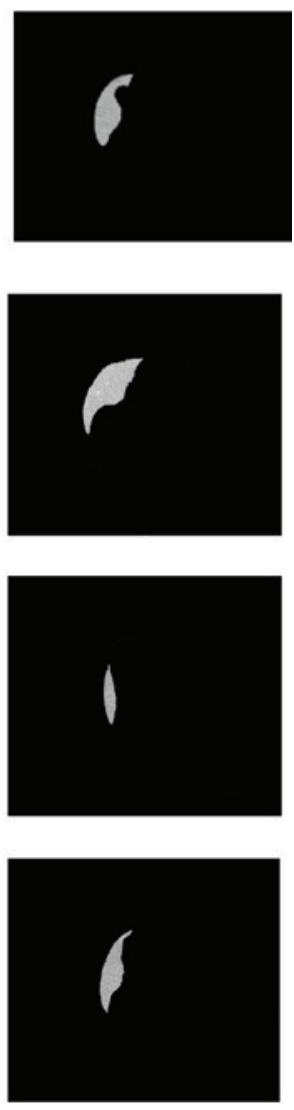

Figure 4: Experimental Results of the Proposed Method and Existing Models.

Columns (A) input image (CT scan image), (B) edge image of the CT scan, (C) speed function of the liver image, (D) liver with boundary, and (E) ground truth image.

\section{Comparison to Existing Methods}

In this section, the performance of the proposed method is compared to the C-V model of Chan et al. [5] with specifications: $\mu=0.1$, number of iterations $=170$, and initial contour position $=[330,310 ; 340,330]$.

Li et al. [17] demonstrated the segmentation process with the following specifications: $\mu=1.0, \varepsilon=1.0$, time step $=0.10, \sigma=4$, initial contour position $=[160,220 ; 190,240]$, number of iterations $=10$. This is called the level set evolution (LSE) model.

Li et al. [16] demonstrated the segmentation process with the following specifications: $\sigma=3.0, \varepsilon=1.0$, $\mu=1.0$, time step $=0.1, \lambda_{1}=1.0, \lambda_{2}=1.0$, number of iterations $=25$, initial contour position in the liver $=[160$, $200 ; 180,200]$. This is called the region-scalable fitting (RSF) model.

The initial contour in the liver section identified in the C-V, LSE, and RSF models and the proposed method are shown in Figure 5A, D, G, and J, respectively. The intermediate results of the C-V, LSE, and RSF models and the proposed method are shown in Figure 5B, E, H, and K, respectively. The final segmentation results of the C-V, LSE, and RSF models and the proposed method are shown in Figure 5C, F, I, and L, respectively.

The liver and its neighboring organs have the same intensity level, and due to more noise, all three existing algorithms have limitations, resulting in the inaccurate detection of the liver section. The proposed methodology fully exploits the intensity distribution information by cropping a random section of the liver and its segmentation resulting in the successful separation of the liver image from its neighboring organs.

In the C-V, LSE, and RSF models, it is necessary to identify the initial contour manually and the number of iterations. These parameters will affect the segmentation results. However, in the proposed method, there 

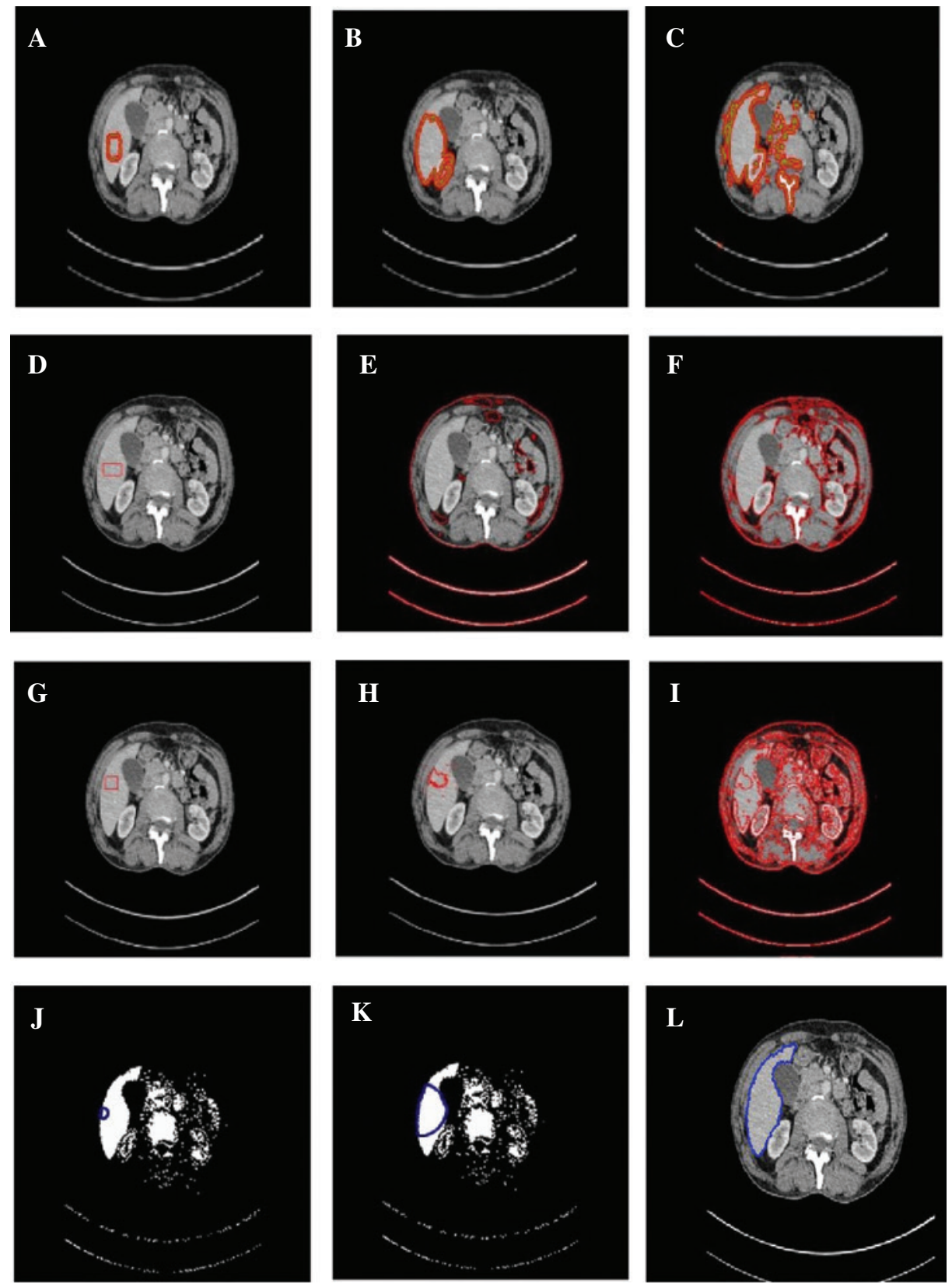

Figure 5: Liver segmentation results comparison between proposed method and existing methods.

(A, D, G, and J) Initial contour in the liver image for the C-V, LSE, and RSF models and the proposed method, respectively; (B, E, $\mathrm{H}$, and $\mathrm{K}$ ) intermediate results for the C-V, LSE, and RSF models and the proposed method, respectively; and (C, F, I, and L) final segmentation results for the C-V, LSE, and RSF models and the proposed method, respectively.

is no need to identify the number of iterations and initial contour manually. Once the complete liver boundary is detected, the results will be displayed on the computer screen. Initial contour is identified without user intervention.

\section{Liver Segmentation Evaluation Metrics}

Image segmentation plays an imperative role in a wide range of applications. Evaluating the competitiveness of the segmentation algorithm for a specified application is obligatory to allow the proper selection of 
segmentation algorithm as well as to fine tune their arguments to achieve acceptable performance. Experimental "disagreement methods" are based on the availability of the reference segmented image, also named the ground truth image. The discrepancy between the segmented image by any algorithm and a ground truth image can be used to gauge the algorithm's performance.

In this paper, distance- and area-based metrics are adopted to evaluate and validate the contour detection process. Segmentations are evaluated with respect to the ground truth image generated under expert supervision.

\subsection{Distance-Based Metrics}

For some segmentation tasks, the delineation of the boundary is critical and is the objective of the segmentation. In these situations, distance-based metrics are essential and used to quantify the distance between the segmentation generated boundary (test boundary) and the "true" boundary. The average symmetric surface distance (ASSD) and maximum symmetric surface distance (MSSD) are two approaches that are normally adopted under distance-based metrics. The "test" boundary is $S_{\mathrm{SEG}}$ and the "true" boundary is $S_{\mathrm{GT}}$

\subsubsection{ASSD}

ASSD is given in millimeters and based on the surface voxels of two segmentations of $S_{\mathrm{SEG}}$ and $S_{\mathrm{GT}}$. Surface voxels are defined with at least one nonobject voxel within their 18 neighbors [12, 20]. For each surface voxel of $S_{\mathrm{SEG}}$, the Euclidean distance to the closest surface voxel of $S_{\mathrm{GT}}$ is calculated using the approximate nearestneighbor technique and stored $[12,20]$. To provide the symmetry, the same process is applied from the surface voxels of $S_{\mathrm{GT}}$ to $S_{\mathrm{SEG}}$. The ASSD is then defined as the average of all calculated distances, which is zero for perfect segmentation [20].

Let $S$ (seg) denote the set of surface voxel of segmentation [20]. The short distance of a voxel v to $S$ (SEG) is defined as

$$
\left.d\left(\underset{S_{\text {SEGeS(seg) }}}{\operatorname{Sin}}\right)\right)=\min |V-S(\mathrm{SEG})|
$$

where $|\cdot|$ denotes the Euclidean distance [20]. The average symmetric surface is then given by

$$
\operatorname{ASSD}\left(S_{\mathrm{SEG}}, S_{\mathrm{GT}}\right)=\frac{1}{\left|S_{\mathrm{SEG}}\right|+\left|S_{\mathrm{GT}}\right|} \star\left\{\sum_{S_{\mathrm{SEG}} \in S(\mathrm{SEG})} d\left(S_{\mathrm{SEG}}, S(\mathrm{GT})\right)+\sum_{S_{\mathrm{GT}} \in S(\mathrm{GT})} d\left(S_{\mathrm{GT}}, S(\mathrm{SEG})\right)\right\}
$$

\subsubsection{MSSD}

MSSD is also given in millimeters and is determined similar to the previous metrics. It is also called the Haudorff distance [12]. Differences between both sets of surface voxels are determined using Euclidean distances and the maximum value yields the MSSD. For perfect segmentation, this distance is 0 [12].

$$
\operatorname{MSSD}=\left(S_{\mathrm{GT}}, S_{\mathrm{SEG}}\right)=\max \left\{\max _{S_{\mathrm{SEG}} \in S(\mathrm{SEG})} d\left(S_{\mathrm{SEG}}, S(\mathrm{GT})\right), \max _{S_{\mathrm{GT}} \in S(\mathrm{SG})} d\left(S_{\mathrm{GT}}, S(\mathrm{SEG})\right)\right\}
$$

MSSD is sensitive to outliers and offers the maximum error. This is required for applications such as surgical planning, where the worst case error is more important than average errors.

\subsection{Area-Based Metrics}

Some segmentation techniques are used to measure the area or volume of the object (e.g. area of the liver and volume of the liver for treatment planning). In these situations, area-based metrics are well known. 
Area-based metrics are used to compare the object enclosed by a segmentation boundary $S_{\mathrm{SEG}}$ and the "true" boundary $S_{\mathrm{G}}$. The two approaches that are used under area-based metrics are true positive fraction (TPF; sensitivity) and false negative fraction (FNF; specificity).

\subsubsection{TPF}

TPF is the area fraction in the "true" segmented boundary $\left(S_{G \mathrm{TT}}\right)$ that is also enclosed by the algorithm segmented boundary $\left(S_{\mathrm{SEG}}\right)$ [8]. For perfect segmentation, TPF is supposed to be 1.

$$
\mathrm{TPF}=\text { Sensitivity }=\frac{S_{\mathrm{SEG}} \bigcap_{\mathrm{GT}}}{S_{\mathrm{GT}}}
$$

\subsubsection{FNF}

FNF is the area fraction enclosed by the "true" boundary $\left(S_{\mathrm{GT}}\right)$ that was missed by the segmentation algorithm $\left(S_{\mathrm{SEG}}\right)$ [8]. For perfect segmentation, FNF is supposed to be 0 .

$$
\mathrm{FNF}=\text { Specificity }=\frac{S_{\mathrm{SEG}}-S_{\mathrm{GT}}}{S_{\mathrm{GT}}}
$$

\section{Discussion}

The proposed design introduces a novel framework for liver segmentation from CT scan images based on NS and FMM. The novel algorithm is proposed to convert an abdominal CT scan image to an NS domain. An NS domain gives an approximate structure of the liver. A new scheme is designed to detect the start points within the liver section, which is the primary requirement of FMM. The start points evolves outwardly to detect the exact liver boundary in the CT scan image. The results obtained are compared to ground truth images.

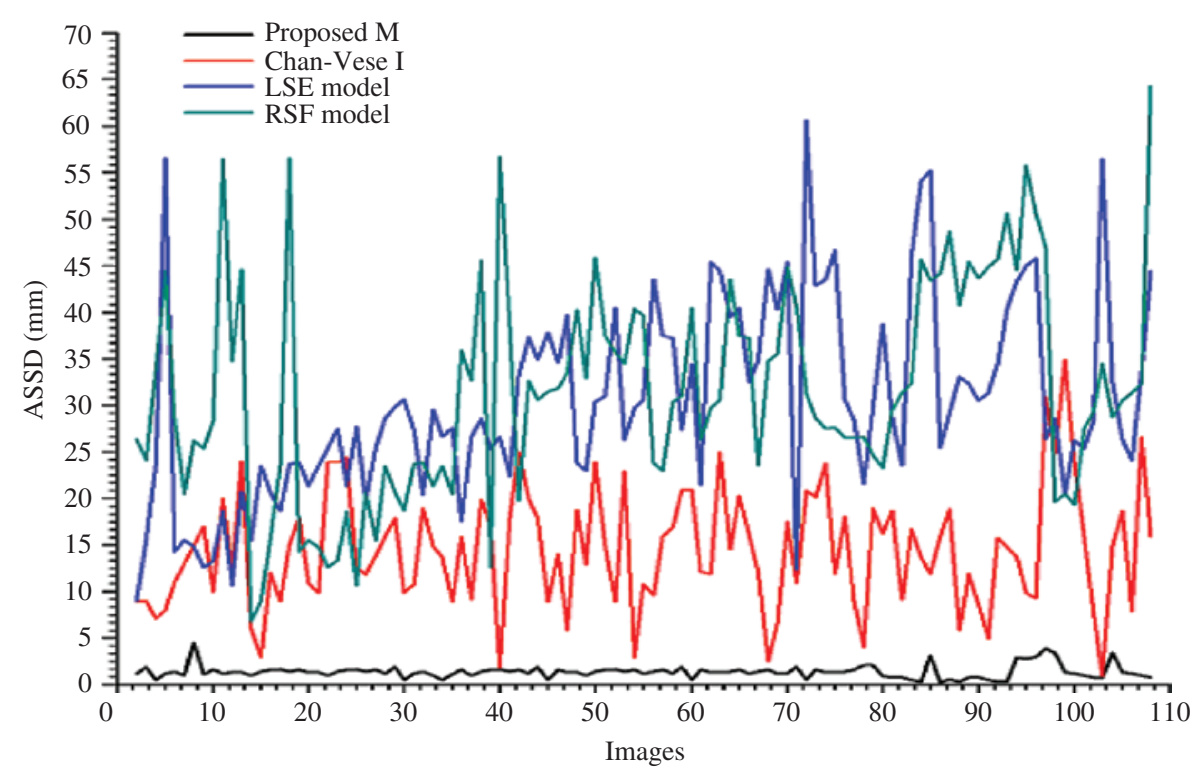

Figure 6: Comparison of Performance Evaluation Results using ASSD. 
To assess the superiority of the proposed method, two types of evaluation metrics are adopted: (1) areabased method (i.e. TPF and FNF) and (2) surface distance-based method (i.e. ASSD and MSSD). The proposed method is compared to the existing method and the comparison results for $108 \mathrm{CT}$ scan images are shown in Figures 6-9. The average ASSD, MSSD, TPF, and FNF for 108 CT scan images for the proposed method and existing algorithm are tabulated in Table 1.

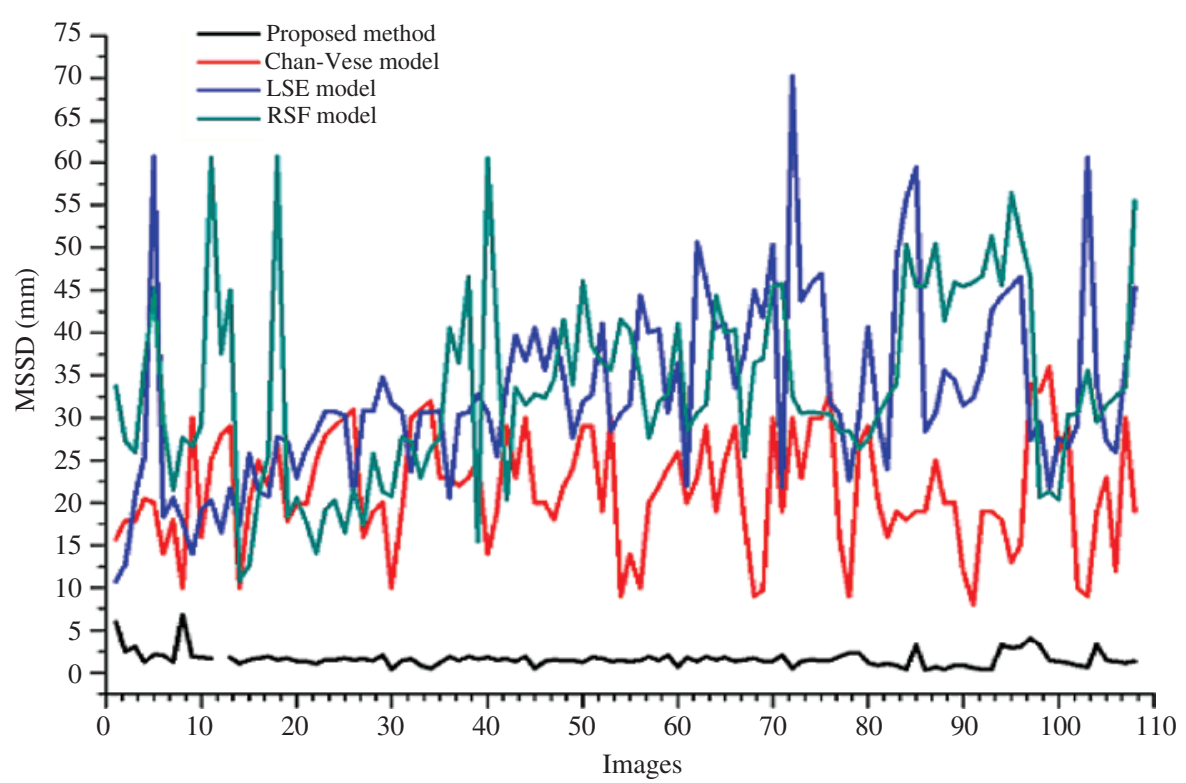

Figure 7: Comparison of Performance Evaluation Results using MSSD.

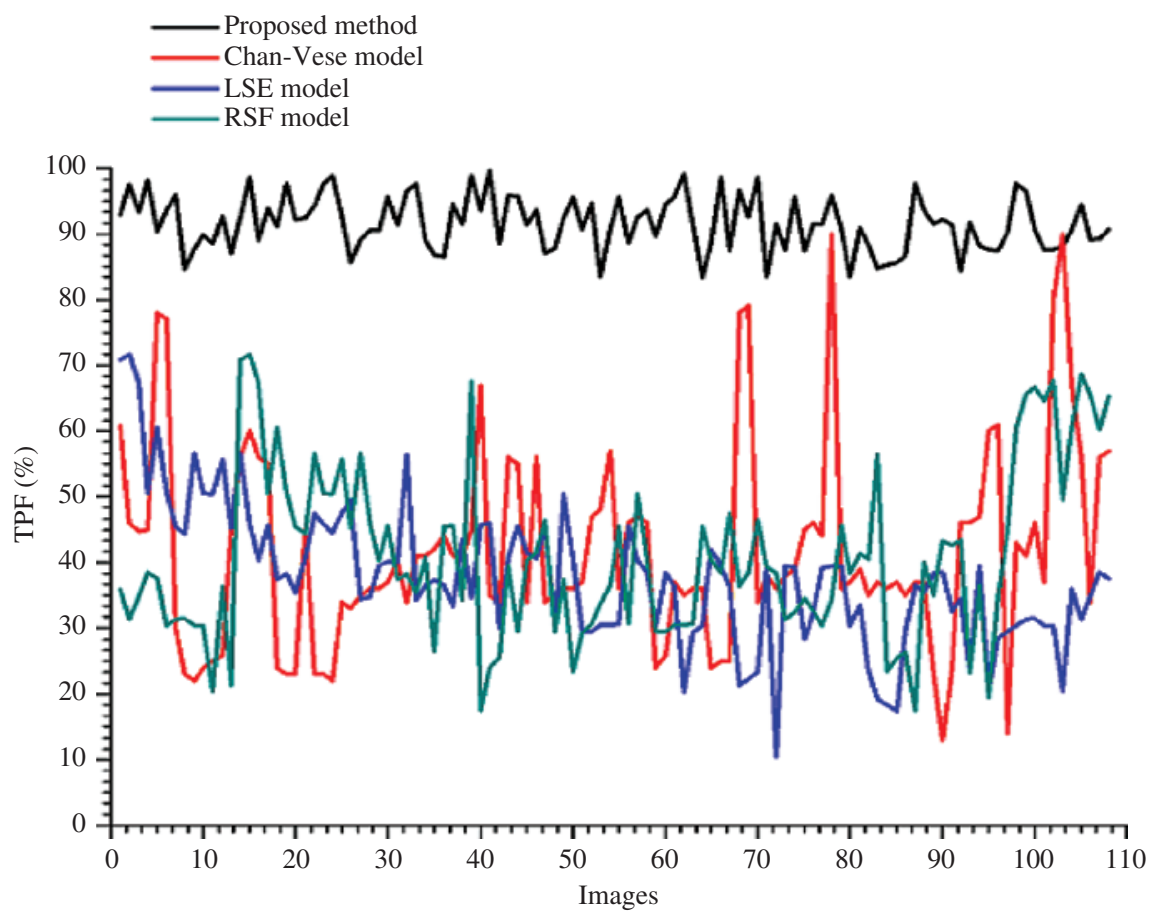

Figure 8: Comparison of Performance Evaluation Results using TPF. 


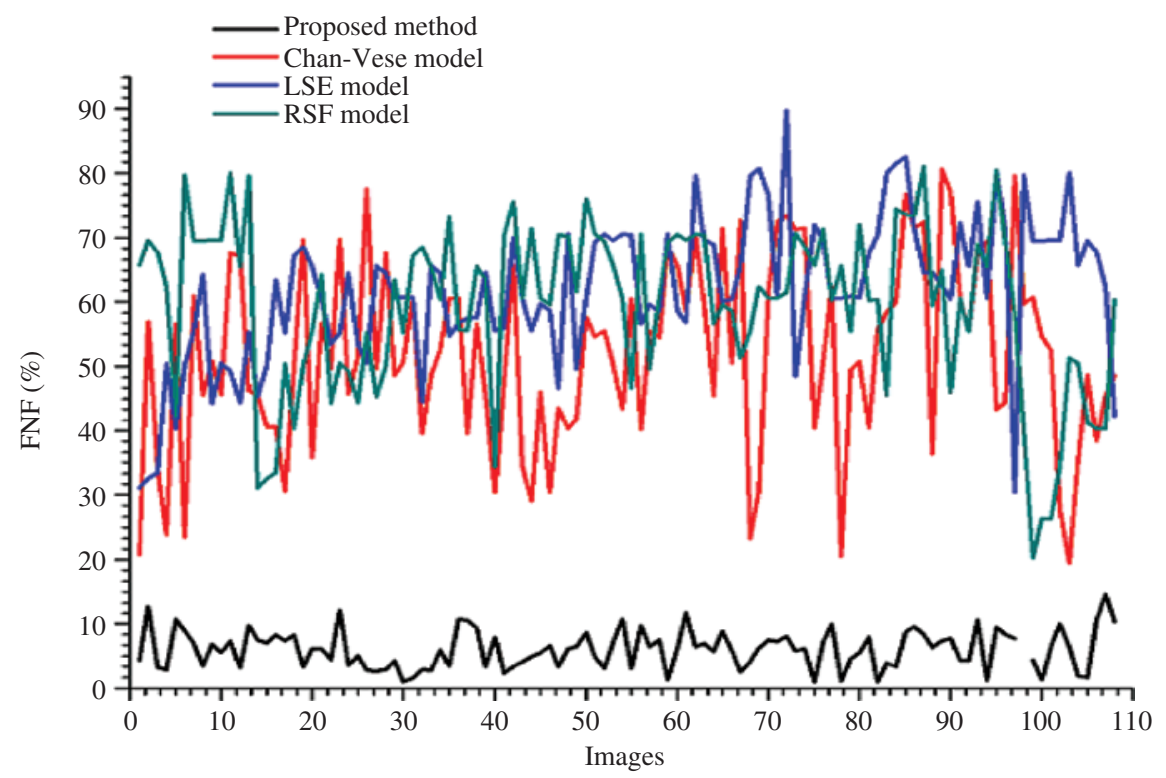

Figure 9: Comparison of Performance Evaluation Results using FNF.

Table 1: Average ASSD, MSSD, TPF and FNF for the Proposed Models and Existing Algorithm.

\begin{tabular}{lrrrr}
\hline & C-V model & LSE model & RSF model & Proposed model \\
\hline ASSD $(\mathrm{mm})$ & $14.3957 \pm 6.4248$ & $30.2300 \pm 10.8651$ & $31.6143 \pm 11.5892$ & $1.3898 \pm 0.7205$ \\
MSSD $(\mathrm{mm})$ & $21.5573 \pm 6.7489$ & $32.7687 \pm 10.7365$ & $33.5736 \pm 10.9680$ & $1.6047 \pm 0.9550$ \\
TPF $(\%)$ & $41.8573 \pm 15.3772$ & $37.8475 \pm 10.7340$ & $41.0407 \pm 13.0454$ & $91.6136 \pm 4.1388$ \\
FNF $(\%)$ & $51.9265 \pm 14.5587$ & $61.8413 \pm 11.3283$ & $59.4688 \pm 12.9994$ & $10.1079 \pm 3.0033$ \\
\hline
\end{tabular}

\section{Conclusions}

This paper designs a liver segmentation method implemented on CT scan images. NS is exploited to remove the neighboring structures of the liver and generated speed function. After locating the start points in speed function automatically, FMM is applied to find an outline of the liver image. This can be used for finding the area and volume of the liver, which helps the physician in liver disease diagnoses and liver transplantation. This can be applied to detect other anatomical structures of the abdomen, such as the kidneys and spleen, with minor modifications.

\section{Bibliography}

[1] N. Akhtar, N. Agarwal and A. Burjwal, K-mean algorithm for image segmentation using neutrosophy, in: Advances in Computing, Communications and Informatics (ICACCI), 2014 International Conference on, IEEE, New Delhi, India, 2014.

[2] N. Al Zaben, N. Madusanka, A. Al Shdefat and H.-K. Choi, Comparison of active contour and fast marching methods of hippocampus segmentation, in: Information and Communication Systems (ICICS), 2015 6th International Conference on, IEEE, Amman, Jordan, 2015.

[3] American Cancer Society, Cancer Facts \& Figures, American Cancer Society, Atlanta, GA, USA, 2010.

[4] R. Bhatt, M. Adjouadi, M. Goryawala, S. A. Gulec and A. J. McGoron, An algorithm for PET tumor volume and activity quantification: without specifying cameras point spread function (PSF), Med. Phys. 39 (2012), 4187-4202.

[5] T. Chan and L. A. Vese, Active contours without edges, IEEE Trans. Image Process. 10 (2001), 266-277. 
[6] Y. Chen, Z. Wang and W. Zhao, Liver segmentation in CT images using Chan-Vese model, in: 1st International Conference on Information Science and Engineering (ICISE2009), Nanjing, China, 2009.

[7] X. Ding, C. Jiang, F. Tian, X. Yan, H. Qi, L. Zhang and Y. Zheng, Fast automated liver delineation from computational tomography angiography, Proc. Comput. Sci. 90 (2016), 87-92.

[8] A. Fenster and B. Chiu, Evaluation of segmentation algorithms for medical imaging, in: Proceedings of the 2005 IEEE Engineering in Medicine and Biology 27th Annual Conference, Shanghai, China, September 1-4, 2005.

[9] J. Ferlay, I. Soerjomataram, R. Dikshit, S. Eser, C. Mathers, M. Rebelo, D. M. Parkin, D. Forman and F. Bray, Cancer incidence and mortality worldwide: sources, methods and major patterns in GLOBOCAN 2012, Int. J. Cancer 136 (2015), e359-e386.

[10] Y. Guo and H. D. Cheng, New neutrosophic approach to image segmentation, Pattern Recognit. 42 (2009), 587-595.

[11] Y. Guo, H. D. Cheng, W. Zhao and Y. Zhang, A novel image segmentation algorithm based on fuzzy c-means algorithm and neutrosophic set, in: Proceeding of the 11th Joint Conference on Information Sciences, Atlantis Press, Shenzhen, China, 2008.

[12] T. Heimann, B. van Ginneken, M. A. Styner, Y. Arzhaeva, V. Aurich, C. Bauer, A. Beck, C. Becker, R. Beichel, G. Bekes, F. Bello, G. Binnig, H. Bischof, A. Bornik, P. Cashman, Y. Chi, A. Cordova, B. M. Dawant, M. Fidrich, J. D. Furst, D. Furukawa and L. Grena, Comparison and evaluation of methods for liver segmentation from CT datasets, IEEE Trans. Med. Imaging 28 (2009), 1251-1265.

[13] A. Heshmati, M. Gholami and A. Rashno, Scheme for unsupervised colour-texture image segmentation using neutrosophic set and non-subsampled contourlet transform, IET Image Process. 10 (2016), 464-473.

[14] A. Jemal, F. Bray, M. M. Center, J. Ferlay, E. Ward and D. Forman, Global cancer statistics, CA Cancer J. Clin. 61 (2011), 69-90.

[15] W. Y. Lau, S. Ho, T. W. T. Leung, M. Chan, R. Ho, P. J. Johnson and A. K. Li, Selective internal radiation therapy for nonresectable hepatocellular carcinoma with intraarterial infusion of 90yttrium microspheres, Int. J. Radiat. Oncol. Biol. Phys. 40 (1998), 583-592.

[16] C. Li, C.-Y. Kao, J. C. Gore and Z. Ding, Minimization of region-scalable fitting energy for image segmentation, IEEE Trans. Image Process. 17 (2008), 1940-1949.

[17] C. Li, R. Huang, Z. Ding, J. C. Gatenby, D. N. Metaxas and J. C. Gore, A level set method for image segmentation in the presence of intensity inhomogeneities with application to MRI, IEEE Trans. Image Process. 20 (2011), 2007-2016.

[18] G. Li, X. Chen, F. Shi, W. Zhu and J. Tian, Automatic liver segmentation based on shape constraints and deformable graph cut in CT images, IEEE Trans. Image Process. 24 (2015), 5315-5329.

[19] F. Lu, F. Wu, P. Hu, Z. Peng and D. Kong, Automatic 3D liver location and segmentation via convolution neural network and graph cut, Int. J. Comput. Assist. Radiol. Surg. 12 (2017), 171-182.

[20] J. Min, M. Powell and K. W. Bowyer, Automated performance evaluation of range image segmentation algorithms, IEEE Trans. Syst. Man Cybern. Pt. B Cybernet. 34 (2004), 263-271.

[21] M. Moghbel, S. Mashohor, R. Mahmud and M. I. Saripan, Automatic liver segmentation on computed tomography using random walkers for treatment planning, EXCLIJ. 15 (2016), 500.

[22] J. Mohan, V. Krishnaveni and Y. Huo, Automated brain tumor segmentation on MR images based on neutrosophic set approach, in: IEEE Sponsored 2nd International Conference on Electronics and Communication System, Othakalmandapam, Coimbatore, India, ICECS, 2015.

[23] R. Murthy, R. Nunez, J. Szklaruk, W. Erwin, D. C. Madoff, S. Gupta, K. Ahrar, M. J. Wallace, A. Cohen, D. M. Coldwell, A. S. Kennedy and M. E. Hicks, Yttrium-90 microsphere therapy for hepatic malignancy: devices, indications, technical considerations, and potential complications, Radiographics 25 (2005), S41-S55.

[24] J. Peng, P. Hu, F. Lu, Z. Peng, D. Kong and H. Zhang, 3D liver segmentation using multiple region appearances and graph cuts, Med. Phys. 42 (2015), 6840-6852.

[25] G. C. Pereira, M. Traughber and R. F. Muzic, The role of imaging in radiation therapy planning: past, present, and future, BioMed Res. Int. 2014 (2014), 231090.

[26] C. Platero and M. C. Tobar, A multiatlas segmentation using graph cuts with applications to liver segmentation in CT scans, Comput. Math. Methods Med. 2014 (2014), 182909.

[27] S. D. Salman AlShaikhli, M. Y. Yang and B. Rosenhahn, 3D automatic liver segmentation using feature-constrained Mahalanobis distance in CT images, Biomed. Tech. Biomed. Eng. 61 (2015), 401-412.

[28] F. Samarandache, A unifying field in logics. Neutrosophic logic, in: Neutrosophy. Neutrosophic set, neutrosophic probability, 3rd ed., American Research Press, Rehoboth, USA, 2003.

[29] G. I. Sayed, M. A. Ali, T. Gaber, A. E. Hassanien and V. Snasel, A hybrid segmentation approach based on neutrosophic sets and modified watershed: a case of abdominal CT Liver parenchyma, in: Computer Engineering Conference (ICENCO), 2015 11th International, Cairo, Egypt, IEEE, 2015.

[30] J. A. Sethian, Level set methods and fast marching methods, Cambridge University Press, Cambridge, England, 1999.

[31] X. Song, M. Cheng, B. Wang and S. Huang, Automatic liver segmentation from CT images using adaptive fast marching method, in Seventh International Conference on Image and Graphics, Quigdao, China, 2013.

[32] R. S. Stubbs, R. J. Cannan and A. W. Mitchell, Selective internal radiation therapy with 90yttrium microspheres for extensive colorectal liver metastases, J. Gastrointest. Surg. 5 (2001), 294-302. 
[33] W. Wu, Z. Zhou, S. Wu and Y. Zhang, Automatic liver segmentation on volumetric CT images using supervoxel-based graph cuts, Comput. Math. Methods Med. 2016 (2016), 1-14.

[34] M. Zhang, Novel approaches to image segmentation based on neutrosophic logic, Utah state university, USA, 2010.

[35] M. Zhang, L. Zhang and H. D. Cheng, A neutrosophic approach to image segmentation based on watershed method, Signal Process. 90 (2010), 1510-1517.

[36] G. Zheng, H.-N. Wang and Y.-L. Li, A tree-like multiphase level set algorithm for image segmentation based on the Chan-Vese model, Acta Electron. Sin. 34 (2006), 1508-1512.

[37] Y. Zheng, D. Ai, P. Zhang, Y. Gao, L. Xia, S. Du, X. Sang and J. Yang, Feature learning based random walk for liver segmentation, PLoS One 11 (2016), 1-17. 